\title{
Molecular Cloning and Characterization of a Putative BnHEC3 Gene in Oilseed Rape (Brassica Napus)
}

\author{
Xiaoli Tan (Corresponding Author) \\ Institute of Life Sciences, Jiangsu University \\ Zhenjiang 212013, China.
}

Tel: 86-511-8291-4326 E-mail: xltan@ujs.edu.cn

Lili Zhang \& Zongwei Xia

Institute of Life Sciences, Jiangsu University

Zhenjiang 212013, China.

E-mail: zhanglily0511@163.com

The research is financed by the National High Technology Research and Development Program (863 projects) of China (No. 2003AA222101) and Postdoctoral Foundation of Jiangsu Province (No. 060101).

\begin{abstract}
HECATE3 (HEC3) is an important transcription factor involved in regulating the carpel and transmitting tract development in Arabidopsis thaliana. The homologous gene of HEC3 in Brassica napus (designed as BnHEC3) was obtained by in silico cloning and rapid amplification of cDNA end (RACE) method. BnHEC3 obtained from cDNA is of $748 \mathrm{bp}$, containing an open reading frame (ORF) of 546bp and coding a peptide of 181 amino acid residues. Sequence alignment revealed that BnHEC3 is highly homologous to HEC3 in Arabidopsis thaliana, especially in bHLH region, which is sharing nearly $100 \%$ identity, and the polygenetic analysis indicated that BnHEC3 belongs to atypical bHLH protein family. RT-PCR results showed that BnHEC3 is mainly expressed in young leave. Southern blotting analysis indicated that BnHEC3 has at least three copies in Brassica napus genome. The transient expression of BnHEC3-sGFP in onion epidermic cells showed that BnHEC3 localized in nuclei. These features implied that BnHEC3 functions as HEC3, and may be involved in the development of female reproductive tissues in Brassica napus.
\end{abstract}

Keywords: Brassica napus, Transmitting tract development, bHLH protein, Transcription factor, Subcellular localization

\section{Introduction}

Properly coordinated development of female reproductive tissues, including stigma, style, septum and transmitting tract, is essential to the successful fertilization. Much of the recent progress has come from studies of model plant Arabidopsis thaliana. Rapeseed (Brassica napus L.) is an important oil and vegetable crop. However, the mechanism of reproductive development in rapeseed remains elusive. The Brassica napus gynoecium, like that of Arabidopsis, is a complex organ specialized for seed production and dispersal. It also arises from two congenitally fused carpels and at maturity consists of an apical stigma for pollen capture, a short intervening style and a large central ovary where ovule and seed development occurs. The ovary is divided into two compartments by the septum (Hill and Lord et al., 1989; Okada et al., 1989; Smyth et al., 1990; Sessions and Zambryski et al., 1995; Gremski et al., 2007). The transmitting tract, a specialized tissue which coincides with the path of pollen tubes in the gynoecium, develops in the center of both the style and the septum to increase fertilization efficiency.

During fertilization, pollen grains germinate on the stigma, and then form a tube to deliver the sperms towards the awaiting ovules. The elongation of the pollen tube during pollination is facilitated by differentiation of the transmitting tract tissue, which is composed of highly secretory cells characterized by an extensive extracellular matrix (ECM). And the transmitting tract cells are also undergoing a program of developmentally controlled cell death (Lennon et al., 1998; 
Wang et al., 1996; Crawford et al., 2007; Gremski et al., 2007). In the absence of proper transmitting tract differentiation, pollen tube growth is limited and fertility is reduced (Crawford et al., 2007; Gremski et al., 2007).

Through studying on the model plant Arabidopsis, a number of genes, including SPATULA (SPT), STYLISH1 (STY1), STYLISH2 (STY2) and ETTIN (ETT), have been proved to be involved in patterning the stigma, style, septum and transmitting tract. In recent years, three new genes, HECATE1 (HEC1), HECATE2 (HEC2) and HECATE3 (HEC3), have been identified as important factors for patterning the transmitting tract, which are necessary in the complex program of gynoecium's development. All of these three genes encode closely related basic helix-loop-helix (bHLH) transcription factors with overlapping function. Loss of HEC function leads to defects in the development of the transmitting tract, septum and stigma, and a decrease in fertility. HEC proteins and SPT, another transcription factor belonging to the bHLH family which plays an important role in the septum and stigma development, are likely to cooperatively interact in controlling development (Gremski et al., 2007).

In this study, we cloned the cDNA of putative BnHEC3 gene (748 bp) from cultivated B. napus cv. Ningyou12 by in silicon cloning and the rapid amplification of cDNA ends (RACE) method (Frohman, 1993). And we showed that BnHEC3 were homologous to HEC3 with nearly 100\% identity in bHLH sequence. Our data suggested that BnHEC3 may function in the process of transmitting tract development in Brassica napus.

\section{Materials and methods}

\subsection{Plant materials and DNA, RNA extractions}

B. napus $c v$. Ningyou12 was used in this study. Roots, stems, leaves, floral buds, and siliques (35d after pollination) were harvested at the indicated stages. RNA and DNA were extracted immediately. Total RNA was isolated with Trizol (Invetrogen) reagent according to the manual. Mature leaves were harvested for genomic DNA extracted with CTAB extraction method.

\subsection{Cloning of the full-length cDNA of BnHEC3}

$50 \mu \mathrm{g}$ total RNA from flower buds of Ningyou 12 was reverse-transcribed into first-strand cDNAs with the PrimeScript Reverse Transcriptase (Takara) in a $50 \mu 1$ reaction volume. For screening of the BnHEC3 from Brassica, the cDNA sequence of HEC3 (NM_121012.1) was used as a query to blast against Brassica database in the TAIR (www.arabidopsis.org), and a putative BnHEC3 EST and a genomic DNA BAC were obtained. The PCR primers (E1/E2, P1/P2 and P3/P4) were designed according to the sequences above.

Primers are as follows:

\section{E1: 5'-CAC TAC TCT TTT CTC CGG AG-3', \\ E2: 5'-AGC TTG ATC TTG CGG CGG-3', P1: 5'-ATC ATC ATA ACG ATC CAA TCG G-3', \\ P2: 5'-ATT GCA CCA TTA CTC ACA CTT TAT-3', P3: 5'-ATG GCC ATG GAC CAA CAC AC-3', \\ P4: 5'-TTA AAT CAG TTC ACC TCC TCC TCC-3'}

The PCR thermocycling conditions were as follows: $94{ }^{\circ} \mathrm{C}$ for $5 \mathrm{~min}$, followed by 35 cycles of $94{ }^{\circ} \mathrm{C}$ for $1 \mathrm{~min}, 60{ }^{\circ} \mathrm{C}$ for $30 \mathrm{sec}$, and $72{ }^{\circ} \mathrm{C}$ for $1 \mathrm{~min}$, and a final extension at $72{ }^{\circ} \mathrm{C}$ for $10 \mathrm{~min}$. The BnHEC3-EST and partial BnHEC3 genomic DNA fragments were purified and cloned into pMD18-T vector (Takara) and sequenced. The 5' and 3' FULL RACE Kits (Takara) were used to clone $5^{\prime}$ and 3 ' flanking sequence of the cDNA.

\subsection{Bioinformatic analysis of BnHEC3}

DNA and amino-acid sequences manipulation were performed with DNAStar 5.0 software. Homology analysis was also performed using Blastx, Genedoc, and BLAST Network Service in TAIR (http://www.arabidopsis.org) and BLAST Network Service in NCBI (http://www.ncbi.nlm.nih.gov/). Expasy database (http://www.expasy.org/) was used to analyze the profile of BnHEC3 protein. The sequences alignment was performed using the program Multalin with the default parameters (http://prodes.toulouse.inra.fr/multalin/multalin.html (Corpet, 1988), and GeneDoc were used for editing sequence alignment. Neighbor-Joining Phylogenetic Tree with bootstrap values was constructed using Mega 3.1.

\subsection{Southern hybridization analysis}

Six restriction enzymes (BamH I, EcoR I, Kpn I, Sac I, Xba I, Xho I) were used for Southern blotting. About $10 \mu \mathrm{g}$ of genomic DNA was digested with 20 unit of restriction enzyme in a final volume of $100 \mu \mathrm{l}$ at $37^{\circ} \mathrm{C}$ for $18 \mathrm{~h}$. The cleaved DNA fragments were run on a $0.8 \%(\mathrm{w} / \mathrm{v})$ agarose gel and transferred to Hybond-N + membranes (Amersham Pharmacia). The BnHEC3-EST (360bp) was amplified with E1/E2 by PCR and purified as a probe for southern blotting hybridization. The membranes were hybridized following the standard protocols (Chomczynski, 1992), and then was washed with $2 \times \mathrm{SSC}$ plus $0.1 \%$ (w/v) SDS at $65^{\circ} \mathrm{C}$ for $30 \mathrm{~min}$ and exposed to X-ray film at $-70^{\circ} \mathrm{C}$. 


\subsection{Analysis of BnHEC3 expression}

Five micrograms of the total RNA isolated from roots, stems, young leaves (before pollination), mature leaves (35 days after pollination), flowers, and pods of the cultivar Ningyou12 were reverse-transcribed into first strand cDNA with the PrimeScript Reverse Transcriptase (Takara), respectively. The first strand cDNA mix was used as template and P3/P4 were used as primers for RT-PCR. The PCR were performed as follows: $94{ }^{\circ} \mathrm{C}$ for $5 \mathrm{~min}$, followed by 35 cycles of $94{ }^{\circ} \mathrm{C}$ for $1 \mathrm{~min}, 60{ }^{\circ} \mathrm{C}$ for $30 \mathrm{sec}$, and $72{ }^{\circ} \mathrm{C}$ for $45 \mathrm{sec}$, and a final extension at $72{ }^{\circ} \mathrm{C}$ for $10 \mathrm{~min}$. BnActin gene amplified with primers $\beta$ - Actin F (5'-ATG GCC GAT GGT GAG GAC ATT C-3') and $\beta$ - Actin R (5'-GGT GCG ACC ACC TTG ATC TTC-3'), was used as an internal control in the experiments.

\subsection{Subcellular localization of BnHEC3}

Motif Scan (http://myhits.isb-sib.ch/cgi-bin/motif_scan) was used to predict the subcellular localization of BnHEC3 and HEC3. sGFP vector, which have a multiple cloning site (MCS) between $35 \mathrm{~S}$ promoter and $s G F P$ gene, was used for constructing BnHEC-sGFP. Briefly, ORF of BnHEC3 without stop coding (TAG) was amplified with two primers containing $\mathrm{Xba}$ I and $\mathrm{BamH}$ I restrict sites respectively as follows: 5'-tct aga ATG GCC ATG GAC CAA C-3' and 5'-gga tcc AAT CAG TTC ACC TCC T-3'. The PCR thermocycling conditions were as follows: $94{ }^{\circ} \mathrm{C}$ for 5 min, followed by 35 cycles of $94{ }^{\circ} \mathrm{C}$ for $1 \mathrm{~min}, 58{ }^{\circ} \mathrm{C}$ for $45 \mathrm{sec}$, and $72{ }^{\circ} \mathrm{C}$ for $1 \mathrm{~min}$, and a final extension at $72{ }^{\circ} \mathrm{C}$ for 10 min. The PCR product was purified, ligated to pMD18-T-vector, and sequenced. Then the correct plasmid was digested with $X b a$ I and $B a m H \mathrm{I}$, and the fragment was gel purified, ligated into the $X b a \mathrm{I} / B a m H$ I predigested sGFP vector to construct BnHEC-sGFP.

The recombination plasmid was transformed into the onion epidermal cells using gene-gun. Then, the onion epidermal cells was cultured under $25^{\circ} \mathrm{C}$ and observed under fluorescence microscope after 16-24 hr.

\section{Results}

\subsection{Cloning and structural analysis of BnHEC3}

HEC3 gene was used as a query to search against the Brassica database, thus an EST and a partial genomic DNA were obtained, which are 360bp (AT002234.1) and 848bp (DU105139) in length, respectively. The EST sequence is same to the corresponding region in the partial BAC sequence, so it is named as the BnHEC3-EST. A complete open reading frame (ORF) indicated by DNAStar was 546bp. Fragments obtained by PCR from cDNA and genomic DNA are uniform, indicating that BnHEC3 has no introns (Fig. 1.).

To get the complete sequence of BnHEC3, RACE method was used. Two sequences with different lengths in 3' ends were obtained by 3' RACE. The length of the longest sequence amplified from cDNA of BnHEC3 is of 748 bp with a $23 \mathrm{bp}$ of incomplete 5'-leader sequence and $219 \mathrm{bp}$ of 3'-untranslated region (Fig. 2.). The putative ORF of $546 \mathrm{bp}$ from BnHEC 3 encodes a peptide of 181 amino acids with a predicted molecular weight of $20.3 \mathrm{kDa}$ and a calculated isoelectric point of 5.88 (Protparam: http:// www.expasy.ch).

Using SMART program (Simple Modular Architecture Research Tool: http:// smart.embl-heidelberg. de), a bHLH domain (amino acid 89-137) was identified in the BnHEC3, and an alanine residue (A) was also found at site 9 within the basic region of the BnHEC3, indicating that BnHEC3 is an atypical bHLH protein, whereas typical bHLH proteins have a glutamic acid residue (E) at that site instead (Fisher and Goding, 1992; Buck and Atchley, 2003; Toledo-Ortiz et al., 2003) (Fig. 2.). The extensive protein sequence similarity between BnHEC3 and HEC3 was a 30 amino acid $\mathrm{N}$-terminal extension of the region of the bHLH domain. This led to BnHEC3 being grouped as a subfamily of bHLH proteins (Heim et al., 2003; Gremski et al., 2007). The conservation was primarily restricted to the bHLH region, and the other regions showed also a high degree of similarity among the bHLH proteins comparing (Fig.3).The bHLH domain of BnHEC3 shared extremely high similarities (nearly 100\%) with that of HEC3 (Fig. 3.). Sequence alignment of the BnHEC3 with HECs and another related bHLH factor INDEHISCENT (IND) revealed that the BnHEC3 also lack a conserved glutamate at position 13 of the basic domain (Fig.3.). This glutamate has been shown to be important for DNA binding (Ellenberger et al., 1994; Ma et al., 1994; Gremski et al., 2007). The phylogenetic tree showed that BnHEC3 belongs to the atypical bHLH proteins group with HEC3, and also strongly supported the high identity between BnHEC3 and HEC3 (Fig. 4.).

\subsection{Genomic structure of the BnHEC3 gene}

The genomic DNA was digested by six restriction enzymes, respectively, and subjected to Southern analysis. The $360 \mathrm{bp}$ BnHEC3-EST was used as a probe. The result was illustrated in Fig. 5. It can be seen that there were three bands (one visible band and two weaker bands) in the first, second and fourth lane. The third lane had only one band but the fifth and sixth lanes had three bands with one weak band and two bright ones. The result of the Southern Blotting suggested that there were at least three copes of BnHEC3 in the genome of B. napus.

\subsection{Analysis of the BnHEC3 expression}

The result of RT-PCR showed that the expression of BnHEC was in young leaves, mature leaves, floral buds and 
siliques rather than in roots or stems, and most strongly in young leaves_(Fig. 6.). Flowers are abnormal organs of the leaves, and they are the essential origin of the fruits in many plants including oilseed rape. The expression profiles of BnHEC3 suggested that BnHEC3 may be involved in regulating the growth and development of leaves and the formation of flower tissues. It may have similar function as $H E C 3$, which can regulate the carpel formation and transmitting tract development in Arabidopsis.

\subsection{Subcellular localization of BnHEC}

As a possible transcription factor, BnHEC3 may be localized in nuclei, however, there was no nuclear localization sequence detected in BnHEC3 by Motif Scan. Whereas, a putative nuclear localization sequence containing Arginine-rich region (RRREISEKIRILKR) was predicted in HEC3 in Arabidopsis. Based on the sequence alignment of HEC3 and BnHEC3, BnHEC3 also contains a motif (RRERISERIRILQR) corresponding to the putative nuclear localization sequence of HEC3, which may be the putative nuclear localization sequence of BnHEC3 (Fig. 2). Further investigate the cellular localization of BnHEC3, transient expression of BnHEC3-sGFP fusion protein in onion epidermal cells was performed. Two kinds of plasmids for transient expression were constructed (Fig.7.A) and transferred into onion epidermal cells respectively. Fluorescence microscopy showed that green fluorescence was observed all over the cells carrying sGFP plasmid, whereas green fluorescence was observed clearly in nuclei of the cells carrying BnHEC3-sGFP plasmid, indicating that BnHEC3 localized in nuclear (Fig. 7.B).

\section{Discussion}

The bHLH transcription factors, such as HEC1, HEC2 and HEC3, play essential roles in development of female reproductive tissues in model plant Arabidopsis thaliana. But their function in Rapeseed (Brassica napus L.) is still unknown. In this study, we successfully identified a novel gene, named as BnHEC3, which may be involved in similar process in Rapeseed.

We obtained the partially complete cDNA of BnHEC3 from Brassica napus using in silico cloning and RACE method. Sequence and phylogenetic analysis indicated that BnHEC3 is highly homologous to HEC3 and belongs to atypical bHLH family. We performed transient expression of BnHEC3-sGFP in onion epidermal cells, and found it is localized in nuclear, which further prove it to be a possible transcription factor as HEC3. Southern blotting of BnHEC3 showed that BnHEC3 has at least three copies, suggesting that BnHEC3 plays an important role in the development process of female reproductive tissues in oilseed rape. Our RT-PCR analysis showed that the BnHEC3 is mainly expressed in young leaves, indicating it may be involved in the specification of floral organs. And it is also expressed in flowers and fruits, suggesting it may have function in regulating the specification of subsequent different cell types in female reproductive tissues.

Before reaching ovules, pollen tubes must travel through several distinct tissues, including the stigma, the stylar transmitting tract and the septum transmitting tract (Crawford et al., 2007; Gremski et al., 2007). Coordinated development of these tissues is crucial for successful fertilization, and the transmitting tract differentiation, is one of the most important process affecting the pollen tube development (Crawford et al., 2007; Gremski et al., 2007). In Arabidopsis, three related bHLH transcription factors, HEC1, HEC2 and HEC3, are identified to be required for this process (Gremski et al., 2007). An RNA-null mutant of HEC3 displayed a loss of fertility and defects in transmitting tract tissue in both septum and style (Gremski et al., 2007). Over-expression of HEC3 led to the ectopic production of stigmatic tissue, consistent with its requirement for stigma development (Gremski et al., 2007).

Therefore, BnHEC3, as the homologous gene of HEC3 in Brassica napus, may also play essential roles in the development of female reproductive tissues. Our study paved the way for subsequent research on BnHEC3 in Brassica napus. By further study on it, we may finally get much more understanding about the fertility mechanism in rapeseed, and the knowledge may be useful to the agricultural production.

\section{Acknowledgments}

This work was supported by the National High Technology Research and Development Program (863 projects) of China (No. 2003AA222101) and Postdoctoral Foundation of Jiangsu Province (No. 060101). We thank Dr. Xinyu Wang for technical helps in BnHEC3 subcellular localization.

\section{References}

Buck, M.J., and Atchley, W.R. (2003). Phylogenetic analysis of plant basic helix-loop-helix proteins. J Mol Evol 56, 742-750.

Chomczynski, P. (1992). One-hour downward alkaline capillary transfer for blotting of DNA and RNA. Analytical Biochemistry 201, 134-139.

Corpet, F. (1988). Multiple sequence alignment with hierarchical clustering. Nucleic Acids Res 16, 10881-10890.

Crawford, B.C.W., Ditta, G. and Yanofsky, M.F. (2007).The NTT Gene is required for transmitting tract development in 
carpels of Arabidopsis thaliana. Curr.Biol.17, 1101-1108.

Ellenberger, T., Fass, D., Arnaud, M. and Harrison, S.C. (1994).Crystal-structure of transcription factor E47-E-box recognition by a basic region helix- loop-helix dimer. Genes Dev.8, 970-980.

Fisher, F., and Goding, C.R. (1992). Single amino acid substitutions alter helix-loop-helix protein specificity for bases flanking the core CANNTG motif. Embo J 11, 4103-4109.

Frohman, M.A. (1993). Rapid amplification of complementary DNA ends for generation of full-length complementary DNAs: thermal RACE. Methods Enzymol 218, 340-356

Grandori, C., Cowley, S.M., James, L.P., and Eisenman, R.N. (2000). The Myc/Max/Mad network and the transcriptional control of cell behavior. Annu Rev Cell Dev Biol 16, 653-699.

Gremski, K., Ditta, G. and Yanofsky, M.F. (2007), The HECATE genes regulate female reproductive tract development in Arabidopsis thaliana, Development 134, 3593-3601

Heim, M. A., Jakoby, M., Werber, M., Martin, C., Weisshaar, B. and Bailey, P. C. (2003). The basic helix-loop-helix transcription factor family in plants:A genome-wide study of protein structure and functional diversity. Mol.Biol.Evol. 20, 735-747.

Hill, J.P. and Lord, E.M. (1989). Floral development in Arabidopsis thaliana: a comparison of the wild type and the homeotic pistillata mutant. Can.J.Bot. 67, 2922-2936.

Lennon, K.A., Roy, S., Hepler, P.K. and Lord, E.M. (1998). The structure of the transmitting tissue of Arabidopsis thaliana (L.) and the path of pollen tube growth. Sexual Plant Reprod.11, 49-59.

Liljegren, S.J., Roeder, A.H., Kempin, S.A., Gremski, K., Ostergaard, L., Guimil, S., Reyes, D.K., and Yanofsky, M.F. (2004). Control of fruit patterning in Arabidopsis by INDEHISCENT. Cell 116, 843-853.

Ma, P.C.M., Rould, M.A., Weintraub, H. and Pabot, C.O. (1994). Crystal structure of MyoD bHLH domain-DNA complex-perspectives on DNA recognition and implications for transcriptional activation. Cell 77, 451-459.

Massari, M.E., and Murre, C. (2000). Helix-loop-helix proteins: regulators of transcription in eucaryotic organisms. Mol Cell Biol 20, 429-440.

Okada, K., Komaki, M.K. and Shimura, Y. (1989). Mutational analysis of pistil structure and development in Arabidopsis thaliana. Cell Diff.Develop. 28, 27-38.

Sessions, R.A. and Zambryski, P.C. (1995). Arabidopsis gynoecium structure in the wild type and in ettin mutants. Development 121, 1519-1532.

Smyth, D.R., Bowman, J.L. and Meyerowitz, E.M. (1990). Early flower development in Arabidopsis. Plant Cell 2, 755-767.

Toledo-Ortiz, G., Huq, E., and Quail, P.H. (2003). The Arabidopsis basic/helix-loop-helix transcription factor family. Plant Cell 15, 1749-1770.

Wang, H., Wu, H.M. and Cheung, A.Y. (1996). Pollination induces mRNA poly(A) tail-shortening and cell deterioration in flower transmitting tissue. Plant J.9, 715-727.

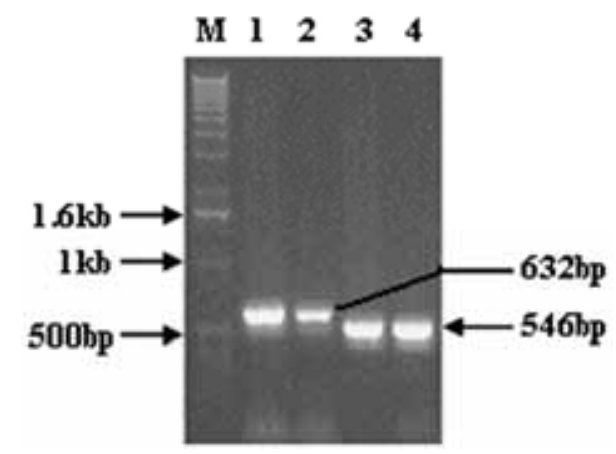

Figure 1. The fragments are amplified by PCR (P1/P2, P3/P4) from cDNA and genomic DNA of BnHEC3, respectively. M: DNA marker; 1 and 3: fragments amplified from genomic DNA of BnHEC3. 2 and 4: fragments amplified from cDNA of BnHEC3. 


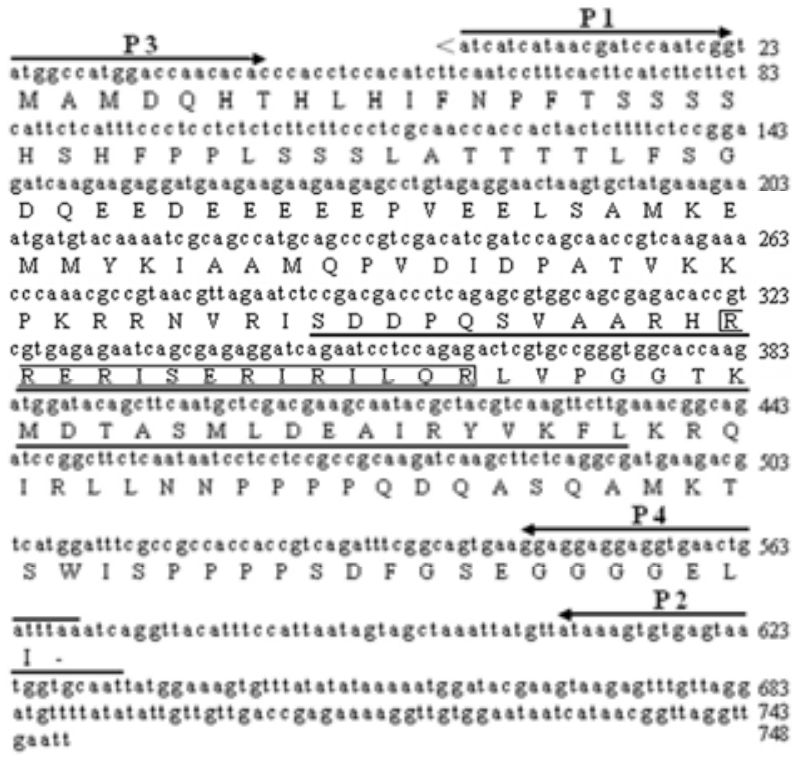

Figure 2. cDNA sequence of the BnHEC3 gene and deduced amino acid sequence.

"<" in front of cDNA sequence showed that the incomplete 5'- end sequence of cDNA of BnHEC3. bHLH domain signature region of BnHEC3 is underlined. A putative nuclear localization sequence was marked with rectangular box. Primers (P1/P2 and P3/P4) were indicated with the arrows.

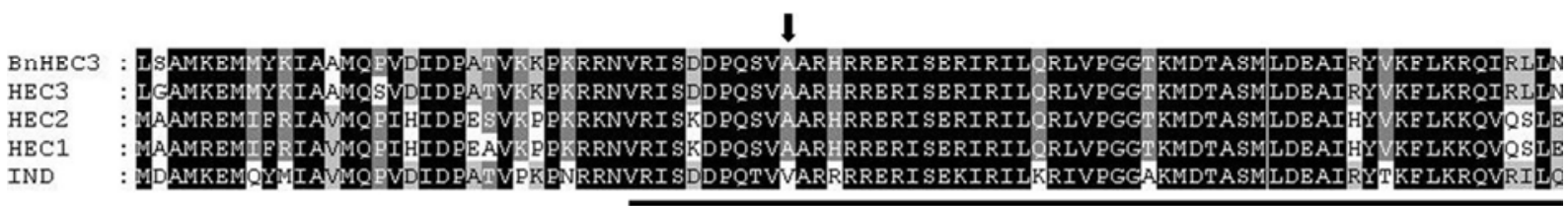

Figure 3. Multiple sequence alignment of the bHLH region from BnHEC3 and other bHLH family proteins from Arabidopsis.

The proteins aligned are: BnHEC3, HECATE3 (HEC3), HECATE2 (HEC2) and HECATE1 (HEC1) (Gremski et al., 2007) and INDEHISCENT (IND) (Liljegren et al., 2004). The black arrow head marks an alanine that replaces the conserved glutamate carried by most other Arabidopsis bHLH proteins. Different grey level represents the different percentage of similarity.

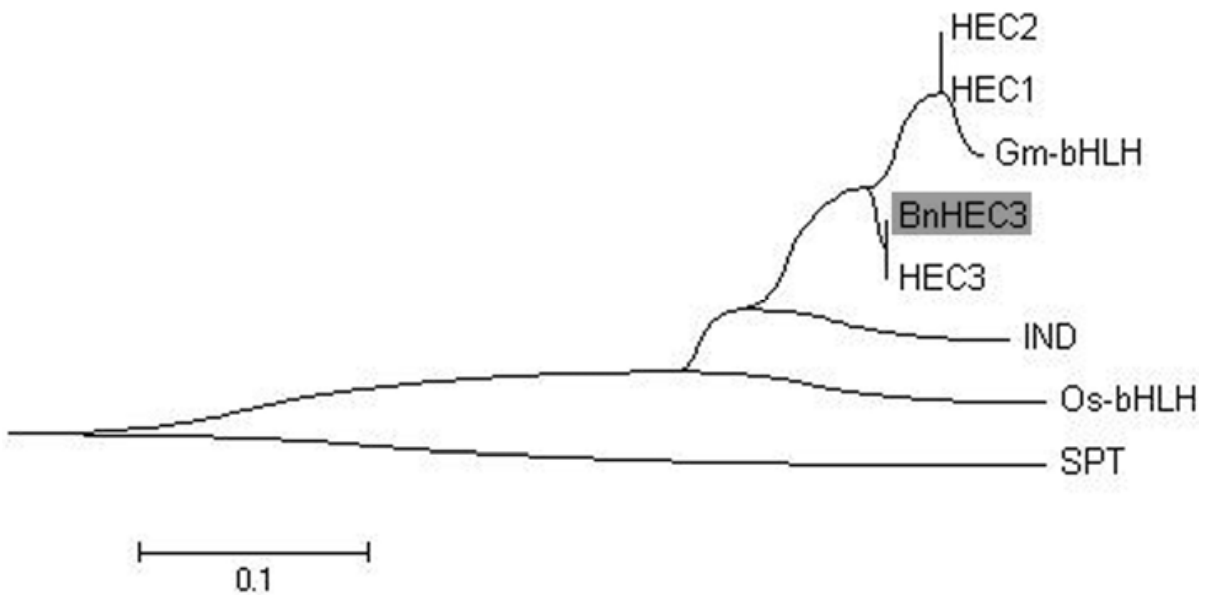

Figure 4. Neighbor-Joining Phylogenetic Tree of the bHLH Domains of bHLH family proteins.

The Phylogenetic Tree showed the phylogenice relationship among the bHLH family proteins, including BnHEC3, HECATE3 (HEC3), HECATE2 (HEC2), HECATE1 (HEC1), SPATULA (SPT), INDEHISCENT (IND), Gm-bHLH (Glycine max-bHLH) and Os-bHLH (Oryza sativa Japonica Group-bHLH). It is obvious that BnHEC3 and HEC3 are highly homologous. 


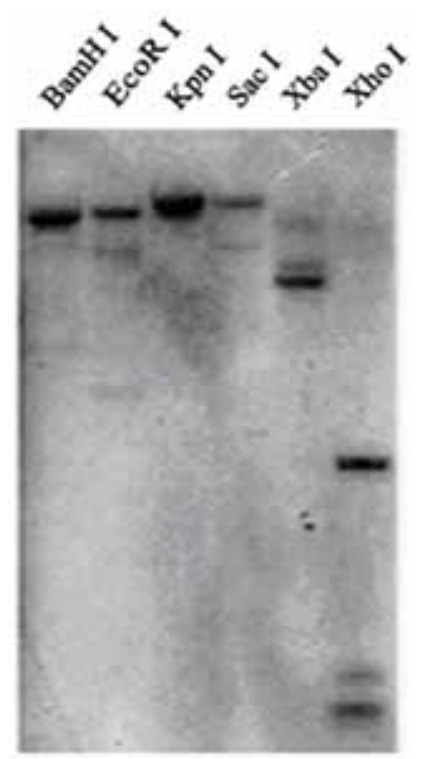

Figure 5. Southern blotting of BnHEC3 gene in Brassica napus genome.

Genomic DNA was digested with BamH I (line 1), EcoR I (line 2), Kpn I (line 3), Sac I (line 4), Xba I (line 5) and Xho I (line 6), respectively, followed by hybridization with the probe of BnHEC3-EST.

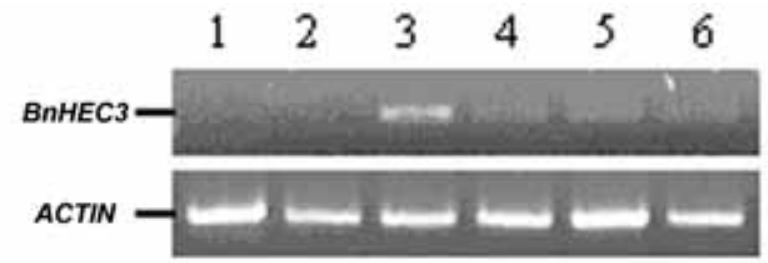

Figure 6. Expression profile of BnHEC3 was analyzed by RT-PCR.

1: roots; 2: stems; 3: young leaves; 4: mature leaves; 5 : floral buds s; 6 : siliques (35d after pollination). $\beta$-ACTIN was used as control.

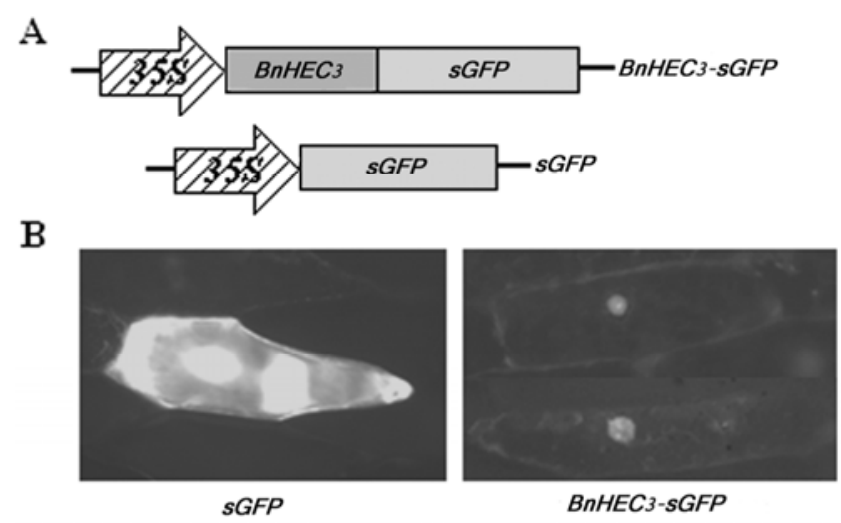

Figure 7. Subcellular localization of BnHEC3 in onion epidemic cell.

(A) Schematic diagram of recombination plasmid. (B) Fluorescent microscopy analysis of subcelluar localization of BnHEC3. It shows the onion epidemic cells that carrying $s G F P$ plasmid (left) and carrying BnHEC3 $-s G F P$ plamid (right). 